\title{
Optimisation of electric arc model parameters based on simplex annealing and genetic algorithms
}

\author{
Tomasz Chmielewski ${ }^{1,{ }^{*},}$ Tomasz Kuczek $^{1}$, and Piotr Oramus ${ }^{1}$ \\ ${ }^{1}$ ABB Corporate Research Center, Starowiślna 13A, 31-038 Krakow, Poland
}

\begin{abstract}
This paper presents the method for obtaining the coefficients of the dynamic Cassie-Mayr electric arc model by means of annealing and genetic optimisation algorithms. The extraction of the coefficients can be obtained by means of the iterative fitting process based on e.g. multiple measurements results. However, this requires a significant effort and can generate significant costs. The approach presented in this paper relies solely on simulations. The methodology used herein consists in finding the match to the maximum produced TRV generated during high-voltage shunt reactor current breaking, for ideal switch and conductance based Cassie-Mayr circuit breaker models. This is done for a given chopping current value assigned to the ideal switch which is used as a target. The arc model coefficients are obtained by means of the optimisation process for various values of the desired chopping current level to be reflected by the Cassie-Mayr conductance-based electric arc model. As a result, an advanced conductance based model can be used for assessment of switching overvoltage. Genetic and simplex annealing algorithms have been selected for optimisation. The models as well as the optimisation process were conducted in EMTP-ATP software using its built-in functionalities. The article presents the error assessment and sample traces.
\end{abstract}

\section{Introduction}

The power system grids are constantly subjected to various switching operations, related to closing and opening of circuit breakers, which require energising or de-energising numerous power systems components, such as transformers, shunt reactors, transmission lines and capacitor banks. Under certain network conditions, such switching operations may result in significant transient overvoltage, which may lead to equipment damage $[1,2$, 3].

This paper is especially dedicated to switching of inductive currents in high voltage networks - it is often of a big concern when it comes to a circuit breaker's capability of successful current interruption when it lags network voltage by $\sim 90^{\circ}$ [4]. Insulation coordination procedures described in IEC 60071 standard series $[1,2]$ provide general guidelines towards the system and circuit breaker modelling. However, there is variety of available models for transient conditions, and they may produce slight changes in the final results, especially in terms of maximum overvoltage peak values. This is a complicated process, and a proper definition of model parameters is important from the perspective of insulation coordination process. In this paper, simulations of $400 \mathrm{kV}$ shunt reactor de-energisation are discussed, since such a scenario reflects hazardous conditions that occur during such circuit breaker opening operation.

Interruption of inductive current may generate highfrequency overvoltage in the situation, when interrupted current is chopped before its natural zero-crossing. When the value of interrupting current is small (a few Amps), the electric arc is unstable, which may lead to chopping the current before its natural zero crossing $[3,5]$. This phenomenon may lead to creating high transient overvoltage because of the oscillations of energy becoming trapped in the oscillatory circuit formed out of the equivalent capacitance and inductance of interrupting the circuit. Making of inductive currents is critical due to the presence of inrush current and possible saturation of equipment magnetic cores. However, this issue is out of the scope of this article. For the process of current interruption, typical waveforms of interrupting current and voltage at the load side present in the system are shown in Figure 1 [6]. Nevertheless, in the visualised case the presence of re-ignitions during switching is not accounted for.

In order to enhance the modelling of the electric-arc applicable for circuit breaker representation and overvoltage estimation, this paper proposes a procedure to obtain the electric arc model coefficients. The proposed method is based on optimisation of Cassie-Mayr electric arc model coefficients values to match the expected overvoltage produced in the idealised system. The methodology is based on multiple simulations run in a specialised software EMTP-ATP with a predefined objective function. The models of electric arc applied in the simulations are based on state-of-the-art equations [57], [8]. Moreover, Cassie-Mayr-based electric arc model was validated by the authors by means of experiments in [10]. The paper presents results for two optimisation

${ }^{*}$ Corresponding author: tomasz.chmielewski@pl.abb.com 
methods i.e. genetic algorithm and simplex annealing algorithm.

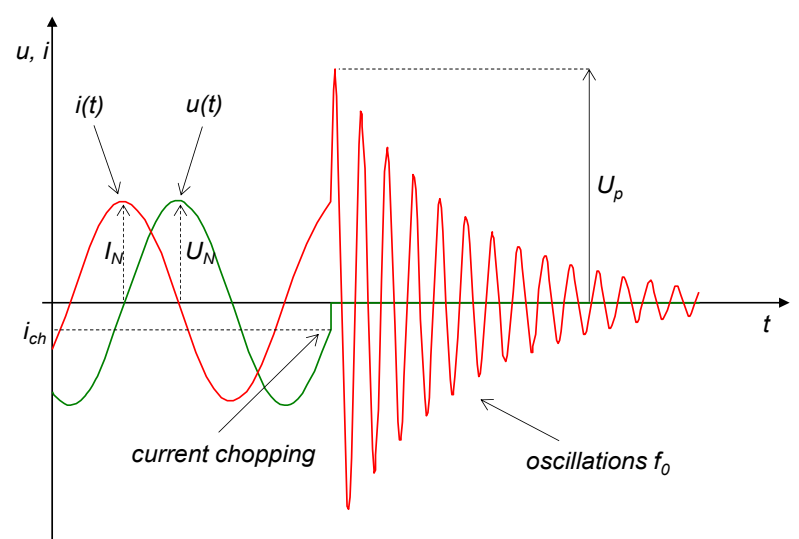

Fig. 1. Waveforms of voltage and current during interruption of inductive current without arc re-ignitions, $i(t)$ - interrupting current $[\mathrm{A}], u(t)$ - voltage at load side $[\mathrm{V}], i_{c h}$ - chopping current $[\mathrm{A}], I_{N}-$ nominal current peak [A], $U_{N}$ - nominal phase voltage $[\mathrm{V}], U_{p}$ - peak voltage after de-energisation $[\mathrm{V}], f_{0}-$ frequency of oscillations $[\mathrm{Hz}]$.

\section{Electric arc models}

In order to simulate switching transient states, different modelling approaches can be used. The standard approach for modelling of current interruption processes implemented e.g. in EMTP-ATP programme is based on the definition of chopping current and ideal switch. In order to compute switching transient states, the value of chopping current is estimated for a considered interrupter by means of the following formula $[7,8]$ :

$$
i_{c h}=k \cdot \sqrt{C}
$$

where: $k$ - chopping number $[\mathrm{A} \cdot \mathrm{F}]^{-0.5}, C$ - equivalent capacitance between contacts of the analysed circuit breaker (capacitance that is seen from terminals of the circuit breaker) $[\mathrm{F}]$.

The value of constant $k$ depends on types of quenching medium applied in the circuit breaker (except for vacuum circuit breakers) [9]. It can be concluded, that an ideal switch model is only a simplified approach that could be used for purposes of insulation coordination analyses. In order to achieve more accurate simulation results, more complex black box models should be used to estimate values of electric arc conductance during switching operation. The black-box model approach controls the conductivity of the electric arc in the function of interrupting current, voltage and time. Hence, the resulting conducted conductivity of electric arc may be introduced to the analysed circuit as a series, incessantly controlled resistance.

One example of black-box approach is Cassie-Mayr implementation. The Cassie equation is relevant to representing interrupting high currents, whereas Mayr formula well reflects phenomena during interruption of small currents (responsible for overvoltage generation). For this reason, only the combination of both equations may produce accurate computation results under different conditions of current interruption. However, unfortunately, coefficients that have to be implemented into equations $\left(\tau_{c}, P_{0}, u_{s}\right.$ and $\left.\tau_{m}\right)$ are strongly dependent on the design of interrupting circuit breaker (its design of contact system, velocity of contacts separation, applied medium for arc quenching etc.). For this reason, the constants must be determined separately for each type of an analysed circuit breaker. The Cassie formula is given by the equation below [8]:

$$
\frac{d g_{c}}{d t}=\frac{1}{\tau_{c}} \cdot\left(\frac{i_{a} u_{a}}{u_{s}^{2}}-g_{c}\right)
$$

where: $g_{c}-$ arc conductance [S], $\tau_{c}$ - time constant of the arc $[\mathrm{s}], u_{s}$ - steady state arc voltage [V], $i_{a}-$ the arc current [A], $u_{a}-$ the arc voltage [V].

The Mayr equation is given by the formula below [11]:

$$
\frac{d g_{m}}{d t}=\frac{1}{\tau_{m}} \cdot\left(\frac{i_{a}^{2}}{P_{0}}-g_{m}\right)
$$

where: $g_{m}-$ the arc conductance $[\mathrm{S}], \tau_{m}-$ the time constant of the arc [s], $P_{0}$ - the steady-state cooling power of the arc [W].

Hence, the total arc resistance is given by $[10,12]$ :

$$
r_{\text {arc }}=\frac{1}{g_{\text {arc }}}=\left(\frac{1}{g_{c}}+\frac{1}{g_{m}}\right)
$$

where: $g_{c}-$ the arc conductance calculated according to the Cassie equation (2) [S], $g_{m}-$ the arc conductance calculated according to the Mayr equation (3) [S].

The typical values of the Cassie-Mayr arc models are as follows: (2) and (3): $\tau_{c}=0.8[\mu \mathrm{s}], \tau_{m}=0.22[\mu \mathrm{s}]$, $P_{0}=8.8[\mathrm{~kW}], u_{s}=2.35[\mathrm{kV}]$ [13]. The electric arc parameters estimation, described in detail in Section 3, was performed only on the Mayr portion and the Cassie equation coefficients were kept as typical values.

\section{Proposed method for Cassie-Mayr electric arc parameters estimation}

The methodology to assess the Cassie-Mayr model parameters is based on the optimisation that minimises the error between the Transient Recovery Overvoltage (TRV) across the ideal switch breaker model and electric arcbased model. The value of the TRV is obtained for a few predefined values of the chopping current using ideal switch. The chopping current is a decisive parameter of the circuit breaker in terms of overvoltage value: the higher the chopping current value, the higher the resulting overvoltage during current interruption. At first, the target value of the overvoltage (for a given chopping current value) has to be calculated from the simulation of the test circuit. Afterwards, this value is used for objective function $f(T R V)$ formulation and minimisation as presented in equations (5) and (6) below.

$$
f(T R V)=\left|T R V_{\text {ideal }}-T R V_{C M}\right|
$$

$\min (f(T R V))$ 
The same objective function is used for two selected optimisation algorithms i.e. Simplex Annealing (SA) and Genetic Algorithm (GA). As explained in the previous section the Cassie-Mayr is described with four coefficients. However, as already mentioned the low current region arc behaviour, hence the one responsible for the overvoltage, is the Mayr part. Therefore, only the Mayr part coefficients $\tau_{m}$ and $P_{0}$ are optimised and Cassie portion coefficients are kept constant with values presented in Section 2. The general description of the proposed procedure is depicted in Figure 2.

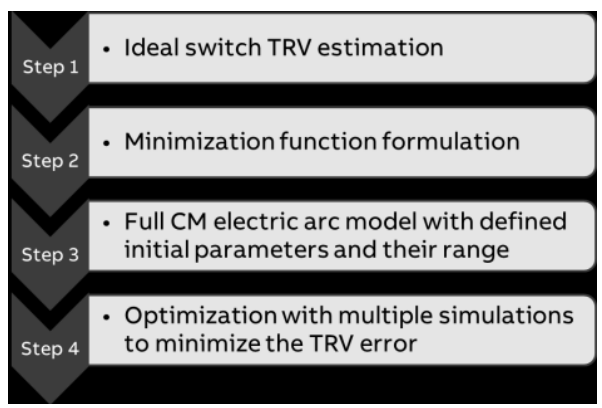

Fig. 2. Proposed procedure for Cassie-Mayr coefficient estimation.

Both considered algorithms are available as part of optimisation tool of EMTP-ATP software. The genetic algorithm can be described by a number of parameters that influence its performance and accuracy. The population is a crucial parameter that depends on the number of variables and problem complexity. For two variables a 100 population size is considered reasonable, bearing in mind that the larger the population size, the more accurate the solution, however simultaneously - the larger computational effort. The selection method for the population can be selected as random, roulette (using cumulative distribution), tournament, stochastic tournament and elitism, yet initial tests have shown that the roulette method yields the best results. The crossover probability, mutation (random) and inversion (from parents) should be low and less than unity but increased for more complex problems. High values can considerably slow down the convergence. When it comes to the simplex annealing, it is also described by population and mutation. However, in this case, the population may be much smaller. The mutation may remain at the same level as in the case of GA. The maximum number of climbs controls how many steps in a negative direction can be accepted by the method (it should be within a range $0-3)$. The parameter $\beta<1$ controls the annealing schedule (temperature reduction), and the parameter ratio controls the annealing schedule when a local minima are found. Both are set at default 0.5 level. $F_{t o l}$ is the convergence criterion which is an additional condition for stopping the optimisation. A more detailed description of applied algorithms may be found in provided references [14, 15]. The optimisation parameters for each algorithm are presented in Section 5.

\section{Description of the studied network}

Test scenarios for SF6 circuit breaker switching studies were conducted in $400 \mathrm{kV}$ grid, as shown in Figure 3.
Selected levels of short circuit power Sk" and X/R ratio are typical for these types of grids.

The transmission line is modelled as a Bergeron type model with a flat horizontal arrangement of phase and shield wires. However, for this type of studies, it is not a critical parameter and has a minor influence on a problem addressed in this paper. The cable that connects the switchgear with transmission line is $50 \mathrm{~m}$ long, and was also modelled as a Bergeron type, with metallic screens bonded on both sides of the cable [2, 3].

The shunt reactor subjected to switching operations is rated at 60 Mvar, and its model includes $60 \mathrm{~kW}$ of total losses and nonlinear magnetisation characteristic with a knee-point voltage at $1.2 U_{n}$ and saturation region slope equal to $30 \%$ [17].

Finally, the circuit breaker included is considered to be $\mathrm{SF}_{6}$ type, and its detailed modelling approach is described in Section 2 of this paper.

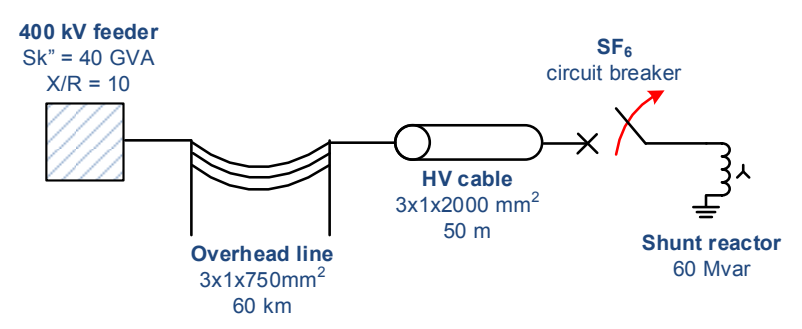

Fig. 3. General diagram of the studied network.

\section{Optimisation settings}

The presented study is based on the electrical network described in the previous section. It bases on the built-in optimisation module in EMTP-ATP software [14-16]. The conducted study included the same electrical parameters but different optimisation of algorithm settings as shown in Table 1 and Table 2.

Table 1. Genetic algorithm settings in optimisation model.

\begin{tabular}{|c|c|}
\hline \multicolumn{2}{|c|}{ Genetic algorithm settings } \\
\hline Population & 100 \\
\hline Resolution & 8-bits \\
\hline Selection & Roulette \\
\hline Crossover_P & 0.98 \\
\hline Inversion_P & 0.1 \\
\hline Mutation_P & 0.01 \\
\hline
\end{tabular}

Table 2. Simplex annealing algorithm settings in optimisation model.

\begin{tabular}{|c|c|}
\hline \multicolumn{2}{|c|}{ Simplex Annealing settings } \\
\hline Population & 4 \\
\hline Mutation_P & 0.01 \\
\hline Max climbs & 2 \\
\hline$F_{\text {tol }}$ & 0.1 \\
\hline ratio & 0.5 \\
\hline$\beta$ & 0.5 \\
\hline
\end{tabular}

In general, eight main scenarios were considered i.e. genetic algorithm and simplex annealing for 15 and 50 iterations with a constant or variable range of optimisation for a given chopping current. The settings of the genetic 
algorithms (except for number of iterations and range of variable) were the same for all considered cases. They are presented in the tables below.

The optimisation procedure is done on two parameters $\tau_{m}$ and $P_{0}$, as already explained. The studies are conducted for various values of chopping current and either constant or variable optimisation range of parameters $\tau_{m}$ and $P_{0}$, as shown in the table below.

Table 3. Optimisation algorithm ranges for the variables at specific chopping current $\left(I_{c h}-\right.$ desired chopping current).

\begin{tabular}{|c|c|c|c|c|}
\hline \multirow{2}{*}{$\begin{array}{l}\mathrm{I}_{\mathrm{ch}} \\
{[\mathrm{A}]}\end{array}$} & \multicolumn{2}{|c|}{ Range $=$ var } & \multicolumn{2}{|c|}{ Range $=$ const } \\
\hline & $P_{0}$ & $\tau_{m}$ & $P_{0}$ & $\tau_{m}$ \\
\hline 1 & $1 \div 1 \mathrm{e} 4$ & $1 e-8 \div 1 e-5$ & \multirow{7}{*}{$\begin{array}{c}1 \mathrm{e} 3 \div \\
3 \mathrm{e} 4\end{array}$} & \multirow{7}{*}{$\begin{array}{c}1 \mathrm{e}-8 \div \\
1 \mathrm{e}-6\end{array}$} \\
\hline 2 & $1 \div 1 \mathrm{e} 4$ & 1 e- $8 \div 1$ e- 6 & & \\
\hline 3 & $1 \mathrm{e} 3 \div 15 \mathrm{e} 3$ & $1 e-10 \div 1 e-7$ & & \\
\hline 5 & $1 \mathrm{e} 3 \div 15 \mathrm{e} 3$ & $1 e-10 \div 1 e-7$ & & \\
\hline 10 & $2 \mathrm{e} 4 \div 3 \mathrm{e} 4$ & $1 \mathrm{e}-8 \div 1 \mathrm{e}-6$ & & \\
\hline 20 & $5 e 4 \div 6 e 4$ & $1 e-10 \div 1 e-6$ & & \\
\hline 30 & $1 \mathrm{e} 5 \div 1.1 \mathrm{e} 5$ & $1 e-10 \div 1 e-7$ & & \\
\hline
\end{tabular}

The following section contains the results of the optimisation procedure based on the presented settings.

\section{Results of the analysis}

This section presents the results of the optimisation procedure based on selected algorithms with different settings. Tables 4 and 5 present the optimal values of $\tau_{m}$ and $P_{0}$ coefficients. The error assessment for both algorithms and all settings is presented in Figure 4 . The mean absolute percentage error was calculated as a main measure of the optimisation methods accuracy, and is displayed in Table 6. The sample traces of voltages and currents are presented in Figure 5 to Figure 8.

Table 4. SA algorithm results of optimisation for various settings.

\begin{tabular}{|c|c|c|c|c|c|}
\hline \multicolumn{6}{|c|}{ Optimisation method : Simplex Annealing } \\
\hline \multirow{3}{*}{$\begin{array}{c}\text { Max } \\
\text { iter. }\end{array}$} & \multirow{3}{*}{$\operatorname{Ich}[\mathrm{A}]$} & \multicolumn{4}{|c|}{ Circuit breaker parameters } \\
\hline & & $P_{0}$ & $\tau_{m}$ & $P_{0}$ & $\tau_{m}$ \\
\hline & & \multicolumn{2}{|c|}{ Range $=$ var } & \multicolumn{2}{|c|}{ Range $=$ const } \\
\hline \multirow{7}{*}{15} & 1 & 4224 & $1.688 \mathrm{E}-06$ & 1000 & $1.000 \mathrm{E}-08$ \\
\hline & 2 & 6745 & $2.874 \mathrm{E}-07$ & 7706 & $3.129 \mathrm{E}-07$ \\
\hline & 3 & 6862 & 2.384E-08 & 30000 & $1.000 \mathrm{E}-06$ \\
\hline & 5 & 13884 & $9.841 \mathrm{E}-08$ & 13519 & $7.885 \mathrm{E}-08$ \\
\hline & 10 & 24981 & $1.000 \mathrm{E}-08$ & 16028 & $1.035 \mathrm{E}-07$ \\
\hline & 20 & 60000 & 8.388E-09 & 16028 & $1.035 \mathrm{E}-07$ \\
\hline & 30 & 107621 & $1.090 \mathrm{E}-08$ & 13519 & $7.885 \mathrm{E}-08$ \\
\hline \multirow{7}{*}{50} & 1 & 1802 & $1.147 \mathrm{E}-07$ & 1000 & $1.000 \mathrm{E}-08$ \\
\hline & 2 & 5791 & $1.656 \mathrm{E}-07$ & 7939 & $3.042 \mathrm{E}-07$ \\
\hline & 3 & 6895 & $2.017 \mathrm{E}-08$ & 30000 & $1.000 \mathrm{E}-06$ \\
\hline & 5 & 13972 & $9.823 \mathrm{E}-08$ & 13145 & $7.196 \mathrm{E}-08$ \\
\hline & 10 & 25386 & $1.000 \mathrm{E}-08$ & 21237 & $1.877 \mathrm{E}-07$ \\
\hline & 20 & 60000 & 4.712E-09 & 2864 & $1.000 \mathrm{E}-08$ \\
\hline & 30 & 103446 & $1.576 \mathrm{E}-09$ & 13519 & $7.885 \mathrm{E}-08$ \\
\hline
\end{tabular}

Table 5. GA results of optimisation for various settings.

\begin{tabular}{|c|c|c|c|c|c|}
\hline \multicolumn{6}{|c|}{ Optimisation method : Genetic Algorithm } \\
\hline \multirow{3}{*}{$\begin{array}{l}\text { Max } \\
\text { iter. }\end{array}$} & \multirow{3}{*}{$\operatorname{Ich}[\mathrm{A}]$} & \multicolumn{4}{|c|}{ Circuit breaker parameters } \\
\hline & & $P_{0}$ & $\tau_{m}$ & $P_{0}$ & $\tau_{m}$ \\
\hline & & \multicolumn{2}{|c|}{ Range $=\mathbf{v a r}$} & \multicolumn{2}{|c|}{ Range $=$ const } \\
\hline \multirow{7}{*}{15} & 1 & 1 & $4.918 \mathrm{E}-08$ & 1910 & $4.565 \mathrm{E}-07$ \\
\hline & 2 & 1962 & 7.942E-07 & 1981 & $6.700 \mathrm{E}-07$ \\
\hline & 3 & 2537 & 7.544E-09 & 2706 & $9.340 \mathrm{E}-07$ \\
\hline & 5 & 5776 & $8.550 \mathrm{E}-08$ & 30000 & 7.600E-08 \\
\hline & 10 & 23020 & $6.506 \mathrm{E}-07$ & 20675 & $6.855 \mathrm{E}-07$ \\
\hline & 20 & 56039 & $1.805 \mathrm{E}-07$ & 12145 & $3.478 \mathrm{E}-07$ \\
\hline & 30 & 107569 & $6.788 \mathrm{E}-08$ & 3388 & $3.905 \mathrm{E}-07$ \\
\hline \multirow{7}{*}{50} & 1 & 8157 & $2.059 \mathrm{E}-07$ & 1341 & $3.866 \mathrm{E}-07$ \\
\hline & 2 & 1609 & $3.633 \mathrm{E}-07$ & 26929 & $4.371 \mathrm{E}-07$ \\
\hline & 3 & 10498 & $6.709 \mathrm{E}-08$ & 12941 & $1.886 \mathrm{E}-07$ \\
\hline & 5 & 4294 & $6.368 \mathrm{E}-09$ & 10780 & $3.051 \mathrm{E}-07$ \\
\hline & 10 & 25255 & $6.506 \mathrm{E}-07$ & 9416 & $3.827 \mathrm{E}-07$ \\
\hline & 20 & 52667 & $2.707 \mathrm{E}-07$ & 22380 & $2.158 \mathrm{E}-07$ \\
\hline & 30 & 104510 & $1.224 \mathrm{E}-08$ & 22039 & $6.195 \mathrm{E}-07$ \\
\hline
\end{tabular}

Table 4 and Table 5 show that the values of $\tau_{m}$ and $P_{0}$ are dependent on the chopping current values, which is expected behaviour. Moreover, it is clear that within one optimisation method the number of iterations influences the results and, despite the fact that in certain cases the ranges of optimised variables overlap, the yielded optimisation result is different due to changes initial conditions. Lastly, it ought to be pinpointed that there may be large discrepancies between the GA and SA methods. This can be further elaborated on the basis of error analysis.

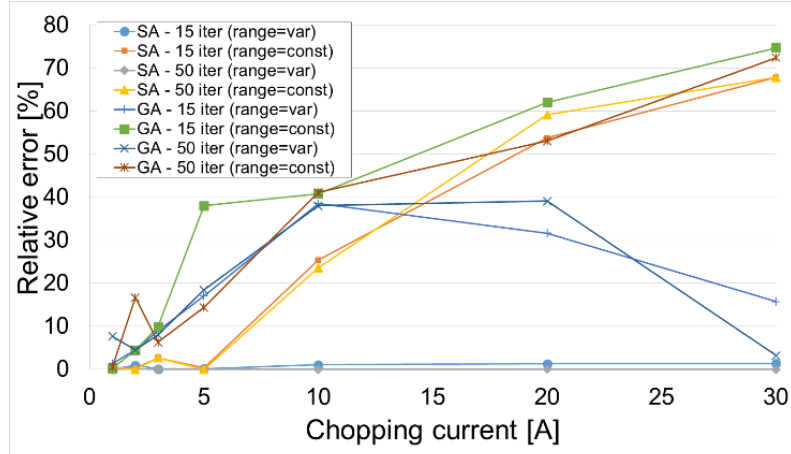

Fig. 4. Absolute relative error summary.

The absolute relative errors between the expected TRV value and the value obtained for optimised parameters of electric arc model are presented in Figure 4. As can be generally observed, the best accuracy is achieved by the SA algorithm with a variable range of optimisation with a maximum error less than $1.4 \%$ for 15 iterations and below $0.7 \%$ for 50 iterations. The GA accuracy is lower especially in the range of high chopping current values. Regardless of the method, the better fitted range of variables tends to improve the accuracy. An 
interesting trend for variable range of optimisation coefficients is observed in the case of larger currents. This can be explained by the better choice of the coefficient range for a given chopping current value. Unfortunately, this range can be either set on the basis of an expert knowledge (as here) or iteratively for consecutive runs of the optimisation procedure.

Table 6. Accuracy of genetic and simplex annealing algorithms for estimation of CM electric arc model coefficients.

\begin{tabular}{|l|c|c|c|c|}
\hline \multirow{2}{*}{\begin{tabular}{l} 
Optimisation $\begin{array}{l}\text { Mean absolute percentage error [\%] } \\
\text { method }\end{array}$ \\
\cline { 2 - 5 }
\end{tabular}} & \multicolumn{2}{|c|}{ Max iter: 15 } & \multicolumn{2}{c|}{ Max iter: 50 } \\
\cline { 2 - 5 } & $\begin{array}{c}\text { Range } \\
=\text { var }\end{array}$ & $\begin{array}{c}\text { Range } \\
=\text { const }\end{array}$ & $\begin{array}{c}\text { Range } \\
=\text { var }\end{array}$ & $\begin{array}{c}\text { Range } \\
=\text { const }\end{array}$ \\
\hline SA & 0.70 & 21.53 & 0.03 & 21.98 \\
\hline GA & 16.83 & 32.90 & 17.00 & 29.22 \\
\hline
\end{tabular}

The summary shown in Table 6, based on the Mean Absolute Percentage Error (MAPE), shows that the best accuracy is obtained for the SA algorithm with variable range different for all chopping currents. It is clear that the main factor improving the results is the range of coefficients used for optimisation. The increased number of iterations did not always result in better accuracy; at least for the considered number of iterations the results are similar in most cases. In general, it can be stated that the simplex annealing method presented better overall accuracy regardless of the number of iterations and the range of the optimised variable. The sample traces of currents and voltages are presented in Figures 6 to 8 below.

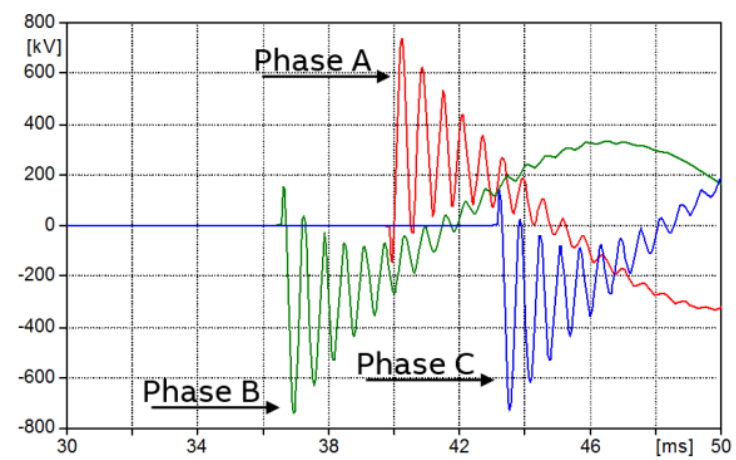

Fig. 5. TRV voltage trace for GA optimisation with target chopping current 3 A (50 iterations).

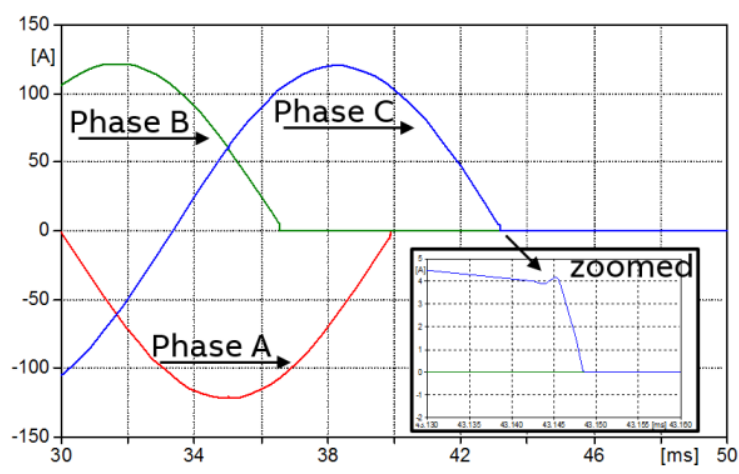

Fig. 6. Interrupted current trace for GA optimisation with target chopping current 3 A (50 iterations).

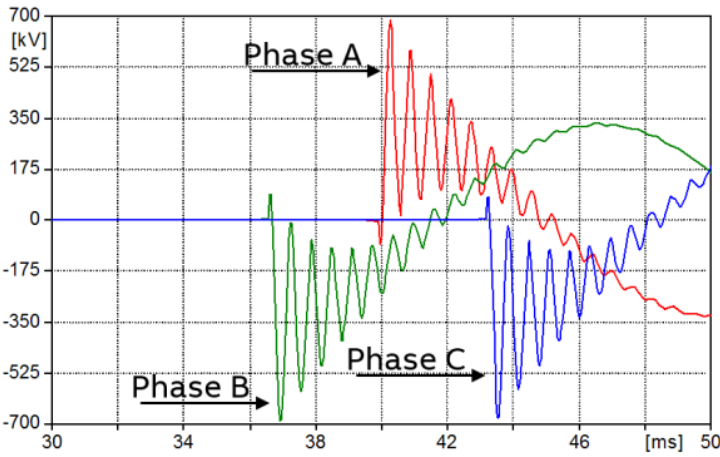

Fig. 7. TRV voltage trace for SA optimisation with target chopping current 3 A (50 iterations).

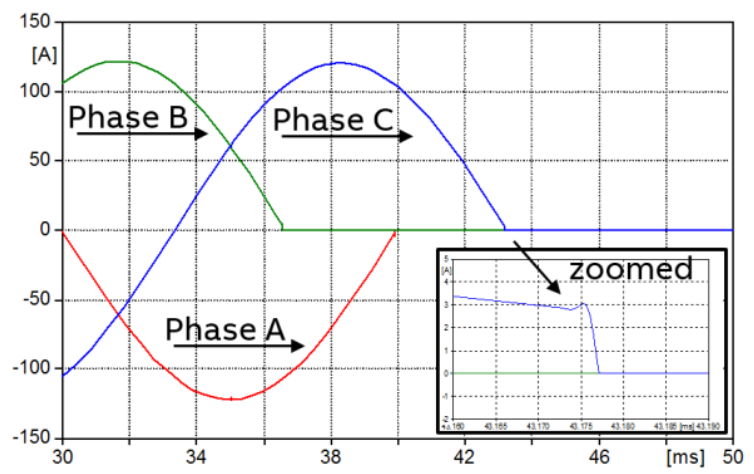

Fig. 8. Interrupted current trace for SA optimisation with target chopping current 3 A (50 iterations).

\section{Summary}

This paper presented the estimation of electric arc model parameter based on proposed optimisation procedure. Two optimisation methods were used for this purpose, namely the genetic algorithm and simplex annealing algorithm. The produced results were based on multiple simulations of the test network with the predefined objective function that was minimised in order to match the target overvoltage.

It has been determined that the considered optimisation algorithms vary in accuracy. The simplex annealing approach yielded better overall performance than genetic algorithm measured by MAPE error. This conclusion is valid regardless of the optimisation method settings and the number of iterations. Moreover, the increased number of iterations has only slightly influenced the accuracy of the optimisation. The largest impact on the accuracy was observed for the appropriate selection of the variable range optimised. Better selection of the variable range resulted in visible accuracy increase.

It was shown that the parameters of electric arc conductance-based models can be obtained in a structured way based on the selected optimisation method and multiple simulations.

\section{References}

1. IEC-60071-1 Insulation co-ordination - Part 1: Definitions, principles and rules (2011) 
2. IEC-60071-4 Insulation co-ordination - Part 4: Computational guide to insulation co-ordination and modelling of electrical networks (2004)

3. CIGRE WG C4.307 Resonance and Ferroresonance in Power Networks (2014)

4. IEEE Std C37.015 - 2009 IEEE Guide for the Application of Shunt Reactor Switching (2009)

5. T. Chmielewski, P. Oramus, M. Szewczyk, T. Kuczek, W. Piasecki, Electr. Pow. Syst. Res. 143, 174-181 (2017)

6. P. Oramus, T. Chmielewski, T. Kuczek, W. Piasecki, M. Szewczyk, Arch. Electr. Eng. 64, 441-448 (2015)

7. G. W. Chang, H. M. Huang, J. H. Lai, IEEE Trans. Power Del. 22, 1533 - 1540 (2007)

8. A. M. Cassie, Proc. of Conf. Int. des Grands Reseaux Electriques a Haute Tension, 1-14 (1932)

9. https://library.e.abb.com/public/433e1e613e67d06ec 1257d0400307817/Buyers\%20Guide\%20HV\%20Li ve $\% 20$ Tank $\% 20$ Circuit $\% 20$ Breakers $\% 20 \mathrm{Ed} \% 206$ en .pdf

10. P. Oramus, T. Chmielewski, T. Kuczek and M. Florkowski, Proc. Conf. PAEE (IEEE, 1-5, 2016)

11. O. Mayr, Arch. Elektrotech. 37, 588-608 (1943)

12. G. Ala, M. lnzerillo, IPST Proc., 493-498 (1999)

13. G. Bizjak, P. Zunko, D. Povh, IEEE Trans. Power Del., 10, 1310 - 1315 (1995)

14. W. H. Press, S. A. Teukolsky, W. T. Vetterling, B. P, Flannery, Numerical recipes, 2nd Ed. (CUP, 1992)

15. http://www.riversoftavg.com/genetic_algorithms.ht $\mathrm{m}$

16. EMTP-ATP Theory Book (Bonneville Power Administration, 1995)

17. IEC-60076-5 Power transformers - Part 5: Ability to withstand short circuit (2006) 